\title{
AN EFFICIENT ANDROID BASED SCHOOL BUS TRACKING SYSTEM
}

\author{
Hemalatha J , Hitaishi K, Mownika \\ V S, Bharat Paul \\ UG Students, \\ Department of Computer Science and \\ Engineering, \\ Nagarjuna College of Engineering \\ and Technology, Devanahalli, \\ Bengaluru, Karnataka, India.
}

\author{
Vivek Sharma S \\ Assistant Professor, Department of \\ Computer Science and Engineering, \\ Nagarjuna College of Engineering \\ and Technology, Devanahalli, \\ Bengaluru, Karnataka, India.
}

\author{
Swetha Vura \\ Assistant Professor, Department of \\ Electronics and Communication \\ Engineering, School of Engineering \\ and Technology, CMR University, \\ Bengaluru, Karnataka, India.
}

\begin{abstract}
These days, guardians are concerned about the school-going youngsters on account of the expanding number of instances of missing students. The parents or guardians must patiently wait for the arrival of school transportation. The use of private vehicles aggravates the current terrible deadlock and many of the guardians consider that school transport is usually dangerous for their children. This task presents the improvement of a school transport observing framework, fit for offering profitable types of assistance through arising advances like Arduino regulator. By using geo location capabilities, our proposed Arduino regulator has the capability to provide the location of children. The proposed system employs the client-server architecture. To save the waiting time of parents, the software developed allows parents to detect the exact location of the school bus via geo location and informs them when the school bus reaches a nearby place. Admin, Driver, and Parents are the three modules included in this framework. The system allocates transportation and its related components space on Google Maps is refreshed by Cloud workers and viewable to administrators. The most up-to-date information of the bus in the form of time and location can be received by parents.
\end{abstract}

\section{Keywords-- GPS, GSM, Arduino regulator}

\section{INTRODUCTION}

Guaranteeing the well-being and keeping school children safe and secure is a major concern for parents. At the point when school-age youngsters travel between school and home, they are prone to being kidnapped. These occurrences make guardians stress over their children. With regards to day-byday undertakings, one of the regular issues is gridlock where the individual vehicles conveying understudies during school hour straightforwardly intensify the adverse consequences of gridlock. This happens because guardians having private vehicles would prefer not to face the challenge of utilizing public vehicles or conventional school transport for schoolgoing kids A new report shows that the number of missing kids, the nation over, expanded by $84 \%$ somewhere in the the range of 2013 and 2016. Innovation can give an extensive answer for these vexing issues. School vehicle Global positioning framework plays a significant part in the wellbeing of younger students. When following a school transport, we propose and trade information. The guardians who do not possess an Arduino-based school transport observing framework, GPS tracks the transport worldwide. The condition of the children and transport gets notified by parents through text message. The data collected by a GPS receiver in the car is transmitted to a Cloud worker [1]. Using GPS coordinates, and mobile device, software allows users to see the spot for bus services as well as monitor the route. By utilizing a Kalman shifting-based forecast calculation, the versatile application additionally decides the appearance seasons of a School Bus. The portable application can consistently refresh the travel times, facilitated with area ID of GPS modules. It utilizes chronicled information and constant data, for the calculation of the appearance times. Two guardians and school authorities will have the documented transport travel history which can be valuable in the future examination, towards guaranteeing the well-being of kids. The system of joining both equipment (GPS beacon) and programming subsystems (Kalman sifting calculation and Android cell phone application) is available in this paper. Plan and execution investigation of the school transport global positioning framework are proposed work centre around. The gathered information is stored in the IoT Cloud stage for preparation to be available in the future.

\section{LITERATURE SURVEY}

The system generally used is Navigation based and manual structure that is anticipated to display the constant space of transportations. This framework requires web affiliation and 


\section{International Journal of Engineering Applied Sciences and Technology, 2021 \\ Vol. 6, Issue 1, ISSN No. 2455-2143, Pages 383-389 \\ Published Online May 2021 in IJEAST (http://www.ijeast.com)}

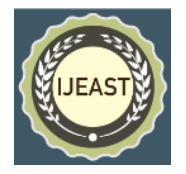

could be a GPS tracker. The infrastructure had a device shown on the cars and gatherer stacks displayed at the vehicle stops. It provides its clients with the required vehicle training and perhaps other information consideration to the entire system that connects the patient's origin to the target Navigation system, which is integrated into each mode of transportation that operates the system. It uses outside gear set-up for its execution. The child safety system developed in [2] employed passive RFID tracking technology because of its wellstructured tracking capabilities, less cost, and easy maintenance. Bus coming in [3] uses a reference point system to recompense for the unavoidable inaccuracies of the locations obtained from the mobile devices. The system in the bus unit is used to recognize when a child boards or leaves the bus. This information is informed to the parent that recognizes which of the children did not board or leave the bus and issues an alert message accordingly [4]. In [5], a dynamic bus timetable is GPS based and a manual system is also designed to display the real time location and timetable of buses which can be useful for any public transport system. A low cost and adaptable security system which is basically based on Arduino with necessary interface to enable Internet and the control of power through Global System for Mobile Communication (GSM) \& Bluetooth module (HC-05) is proposed in [6]. A mobile bus tracking system is presented in [7] to track highway buses using a flexible and a reliable system with the help of GSM and GPS. In [8], a real-time bus tracking system for the public transport is presented to reduce the waiting time of students at bus stops. SMS based tracking architecture with RFID tags is recommended in [9] to reduce the number of kidnapping and road accident cases.

\section{EXISTING SYSTEM}

The current frameworks are utilized for following, observing, and alarming for the vehicle, truck, freight, transport, and bicycle. The system requires the framework to know the continuous area of the vehicle for security reasons and to keep away from burglary of vehicle and caution the proprietor. The payload utilizes a constant observing framework. In case of transportation, it is utilized for constant following of transport, anticipate appearance time, and send data to the base station. The public transportation utilizes following and checking of the transport for appearance time expectation of transport on the individual stops. a few highlights are normal. For example, GPS is utilized for the following purposes, while RFID is utilized for verification. To move data to guardians, hardly any normal mediums have been utilized, H. Ben et al. [1], introduced a screen system inside the vehicle which shows the students are in a safe condition inside the vehicle. The system was a combination of GPRS (General Packet Radio Service) progressions, RFID, and GPS (Global Positioning System). It was displayed using illuminating text on Arduino controller. The current structures can work to perceive the space of the vehicle using GPS similarly as to tell the gatekeepers when the students reach to the vehicle. RFID names are inspected to achieve the target. Each student has RFID card installed into his school gear. At whatever point a student goes into or exits from the transport, the time, date, and game plan are recorded for each customer, and the information is then sent to a security authority. The whole collaboration is personalized, necessitating no activity from the driver or student.

\section{PROPOSED SYSTEM}

The proposed system gives the specific location of the transport, and it gives one greater facility called "Caution facility" to the parents. In this facility parents can add one or more markup points that is the previous location of the transport from where the bus stops and when the bus starts moving from that markup point, then consequently alert is ringing on the guardian's portable device.

It likewise gives the accompanying highlights:

- It gives the driver's contact number, bus route and stops and the specific bus number.

- Manager, Transporter, Authorized Students, and School Board Authenticity.

- In case of an emergency, the administrator has the authority to send text messages to the scheduled driver and student.

\section{SYSTEM DESCRIPTION}

\section{SYSTEM ARCHITECTURE}

The substructure which uses two Arduino applications, one is for overseer and driver which is adaptable application, and another versatile application for customer. Framework grants chairman to incorporate where the framework is present for another driver and in that driver's username, a secret word is created and is taken care inside the framework. The Android application is installed in every driver's android phone, and when the driver starts his journey, he will login to that application. As soon as he $\log$ in, the information base like GPS Region will be sent and taken care. And when the driver signs into that application, it will store the information of GPS is followed into dataset and standard spans. When the driver signs out from the application again, the GPS region will be sent to the framework. The parents can get web interface of the organization which gives customer name and secret key to the parents to follow the vehicle. Fig. 1 shows the school bus tracking system. 


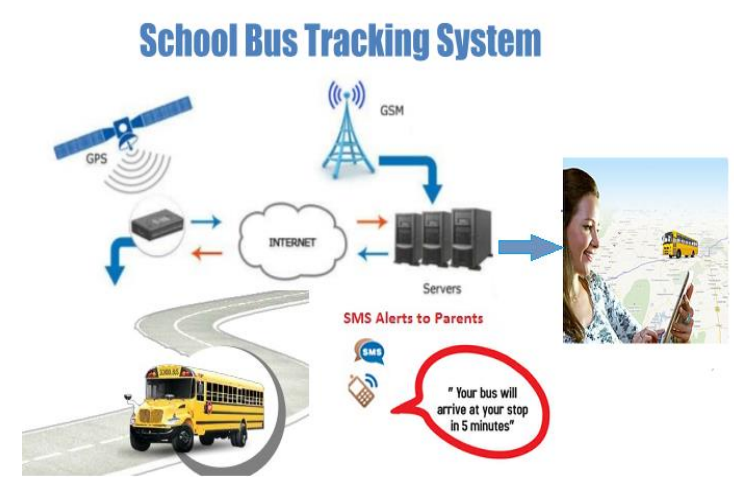

Fig. 1. School bus tracking system

Driver can change the focal points besides, he can review the transport components, focal points of the parents, and he can open the components of the driver. As soon as the bus starts from the school, parents whose children are enrolled for that bus will gets a notification "Bus Starts from the School" and when the bus again reaches the school, they will get another notification "Bus Reaches to School". It gives one more facility called "Ready Facility", in that parents can add markup point and when the vehicle moves from the markup at that point normally, an alert rings on gatekeeper's device.

\section{MODULE DESCRIPTION}

\section{1) Administrator Module}

This section is for the vehicle director, he has all the information related to vehicle drivers, path, and halts. In case of any emergency, if the administrator wants to convey any message to the driver, he has the access to send that to the driver at any time. We are utilizing Way2SMS administrations to achieve this. Fig. 2 shows the admin page features and advantages.

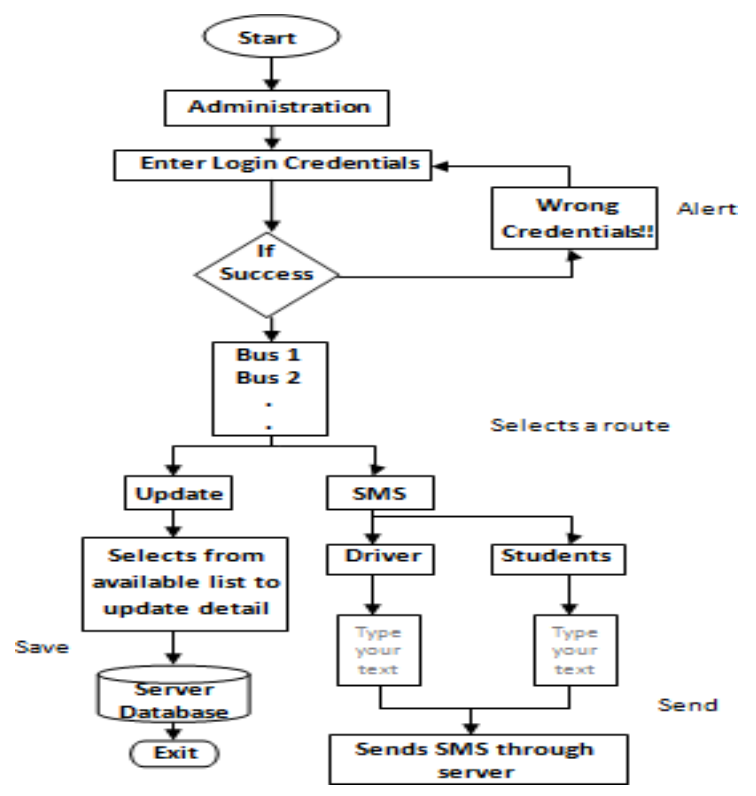

Fig. 2. Flow Diagram for Administration Module

\section{3) Driver Module}

Driver must enter the bus number, route id, and mobile number to begin, the driver must insert valid credentials provided by the organization's vehicle director. The driver can access the pathways and stop the location from the app that is saved on the server. The driver's primary responsibility is to start and stop the vehicle. When the driver starts the transportation, the vehicle begins to move, and when the driver stops the transportation, their respective movements are saved in the server. Below Fig. 3 shows the driver page login and their features.

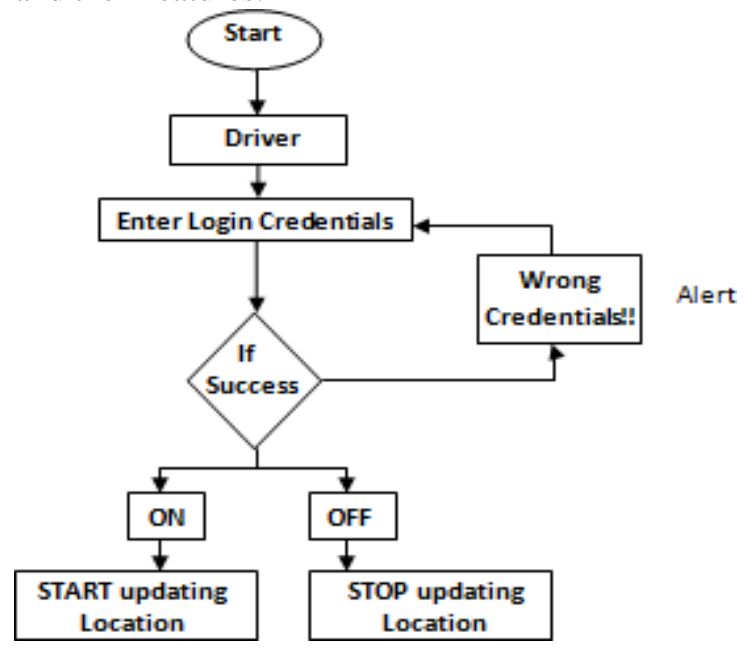

Fig. 3.Flow Diagram for Driver Module 


\section{4) Parent Module}

With tapping on the Live Location choice on the home action, the action for Live Location will be dispatched. There will be text box for submitting current date to show current area of transport on that specific day. One can see the area data of any customer if he wants to login to the application, he needs to enter the mobile number which is registered and the portable number. To look about the moving vehicle in the search bar, customer needs to enter the vehicle number. When he enters all the numbers, the map will show the current region of the vehicle. Likewise, parents can get ready warning when the vehicle arrived at the closest stop. When the application gets dispatched from the customer to choose the courses from the worker and ties to the spinner. At that point when the customer chooses a course, comparing prevents are gotten from the worker and to the spinner for the customer to choose If the customer chooses "Get Location" at that point the area subtleties of the transport for that course is brought. Assuming the customer chooses "Show the area of the Map" the vehicle on the area which is on the guide will be shown. Fig. 4 shows the customer's page for login.

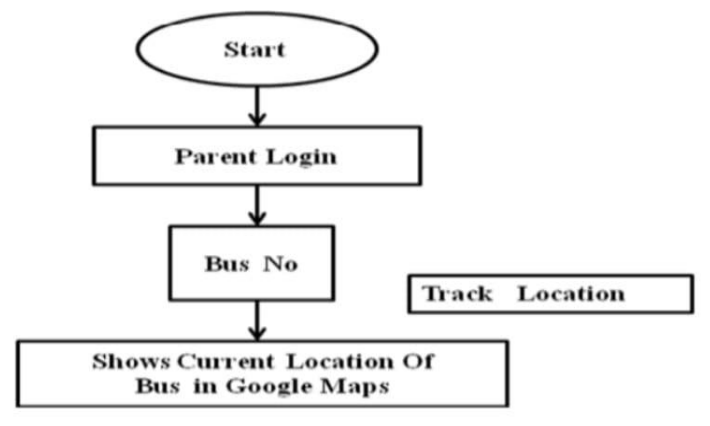

Fig. 4. Flow Diagram for Parent Module

\section{SYSTEM REQUIREMENTS}

SOFTWARE REQUIREMENTS:

- Windows XP, Windows 7(ultimate, enterprise)

- Arduino IDE

- Embedded C

- Blynk App

HARDWARE REQUIREMENTS:

- Processor - i3

- Hard Disk - 5 GB

- Memory - 1GB RAM

- Android Phone with kit-kat and higher.

- Node MCU

- GPS

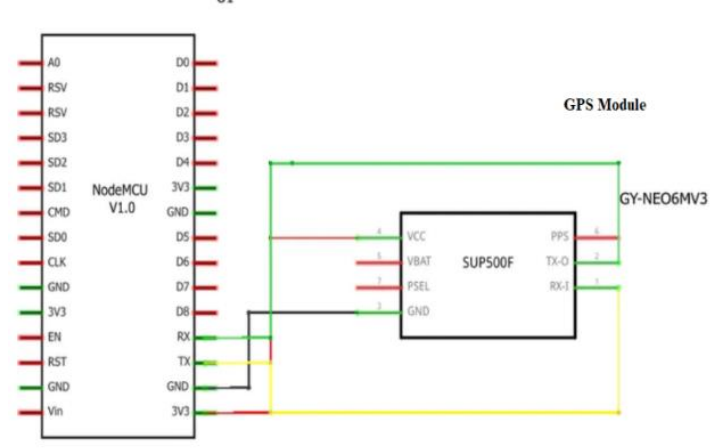

Fig. 5. System Schematic Diagram

\section{METHODOLOGY}

The Android-based School Bus worldwide locating architecture is also an IOT-based project that can inform passengers including the present location of its transportation using a post-mobile App named Blynk. Blynk app permits us to predict both distance and time of the transport reach and travel examples to the stop; It assists guardians with following their children's vehicle position through network access. After obtaining longitude and latitude, coordinates of the moving bus location, Node MCU can obtain information via a device Blynk server. For getting the GPS location of the school bus GPS module is interfaced with the Node MCU development board. Fig. 5 shows interfacing of GPS module with Node MCU development board. If GPS module gets 5V supply, it continuously sends string which contains longitude and latitude of location where GPS module is physically present. As the Google map is maintained within the vehicle, then that will communicate the length and breadth of the school bus on its ideas effectively, which is connected to the NODEMCU circuit, as shown in the diagram. String which is available on serial port is divided into various fields, each field dedicated to specific parameter. After reading string from GPS module Node MCU stores it and sends it to the cloud.

\section{IMPLEMENTATION AND RESULTS}

First, we must create a project on Blynk app (Fig. 6), then select the name of the GPS tracker and board node MCU (Fig. 7). We then receive an authorization token to our mail (Fig. 8). We need to click on the plus sign (Fig. 9) to be able to select the map (Fig. 10). The interface is displayed as in Fig. 11 where the map setting is chosen (Fig. 12) to be able to select the Virtual pin (Fig.13). 
International Journal of Engineering Applied Sciences and Technology, 2021

Vol. 6, Issue 1, ISSN No. 2455-2143, Pages 383-389

Published Online May 2021 in IJEAST (http://www.ijeast.com)

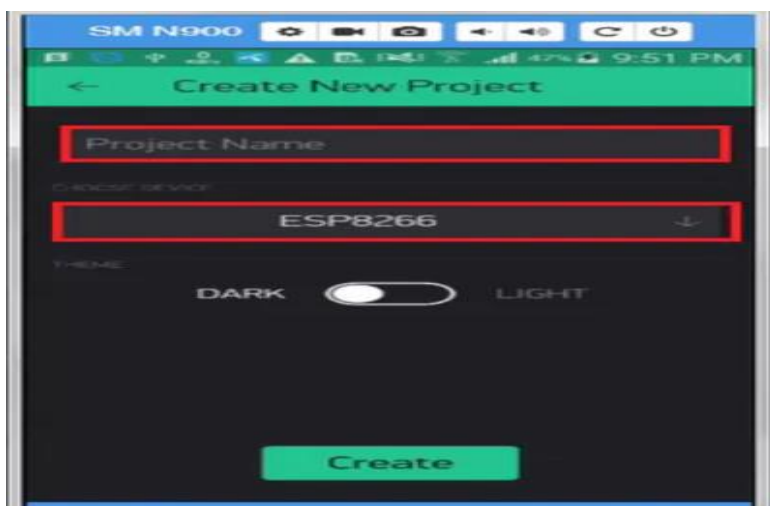

Fig. 6. Click on New Project Then Select Name and Device

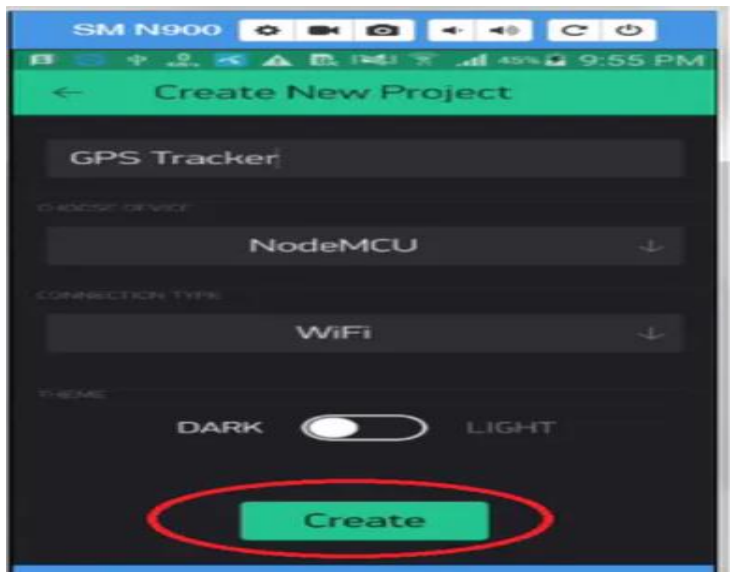

Fig. 7. Select Name GPS Tracker and Board Node MCU then click Create.

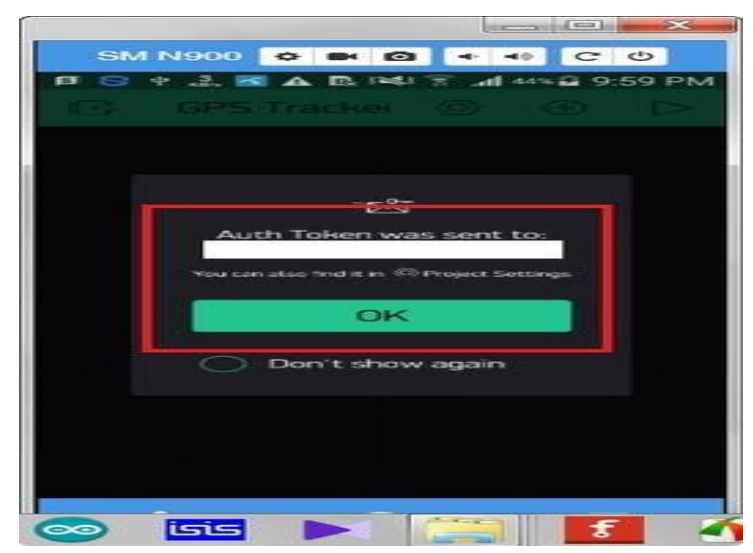

Fig. 8. An Authorization Token is sent to the mail address

Security and mobile app are given huge importance. Hence, choosing the right authorization method is important thing when creating the web service. Therefore, to ensure data transmission security Auth Token is used. Authorization server issues a random token with credentials to the users for authorization request. Whether the passenger is allowed to use the entity, explicit activities can be determined by the data which is sent to the content administrator.

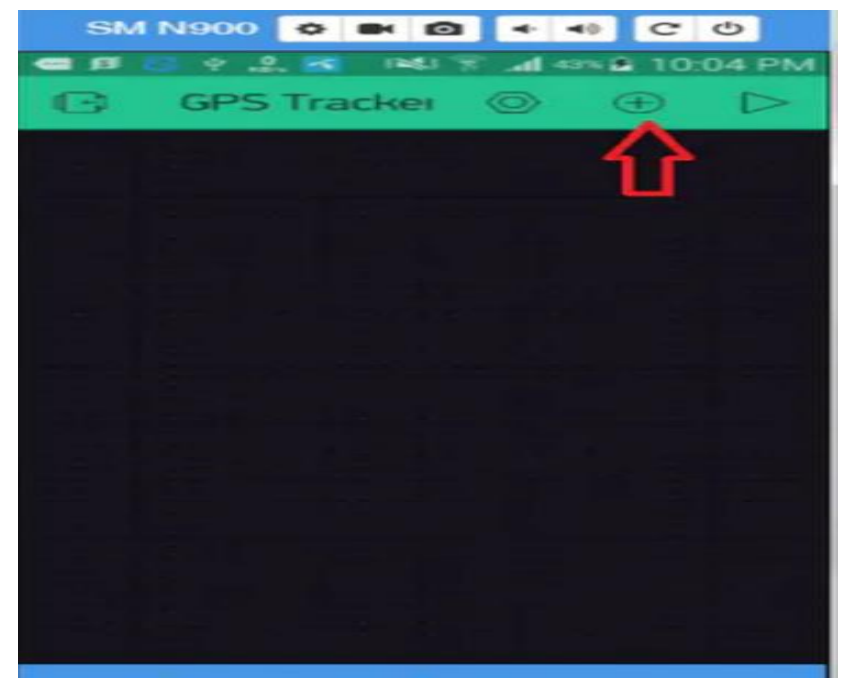

Fig. 9. click on plus sign

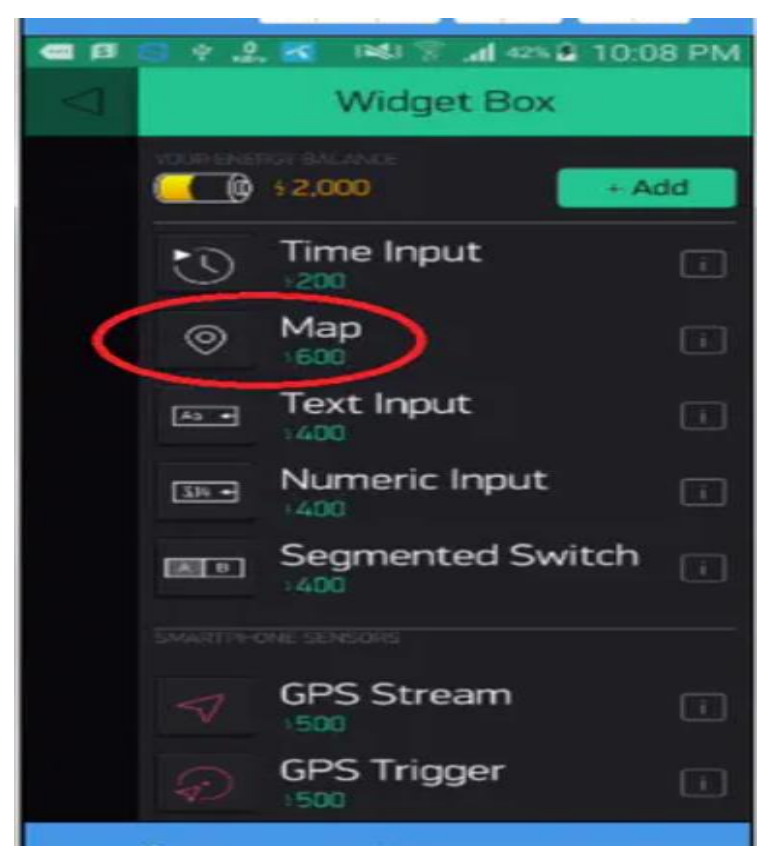

Fig. 10. Select map 
International Journal of Engineering Applied Sciences and Technology, 2021

Vol. 6, Issue 1, ISSN No. 2455-2143, Pages 383-389

Published Online May 2021 in IJEAST (http://www.ijeast.com)

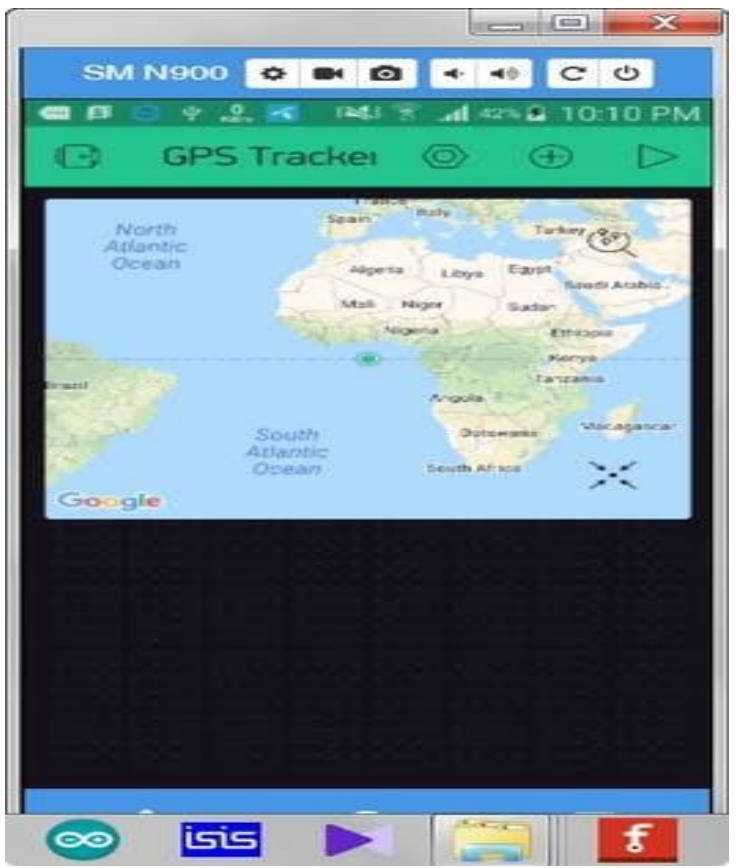

Fig. 11. After selecting map, the interface is displayed

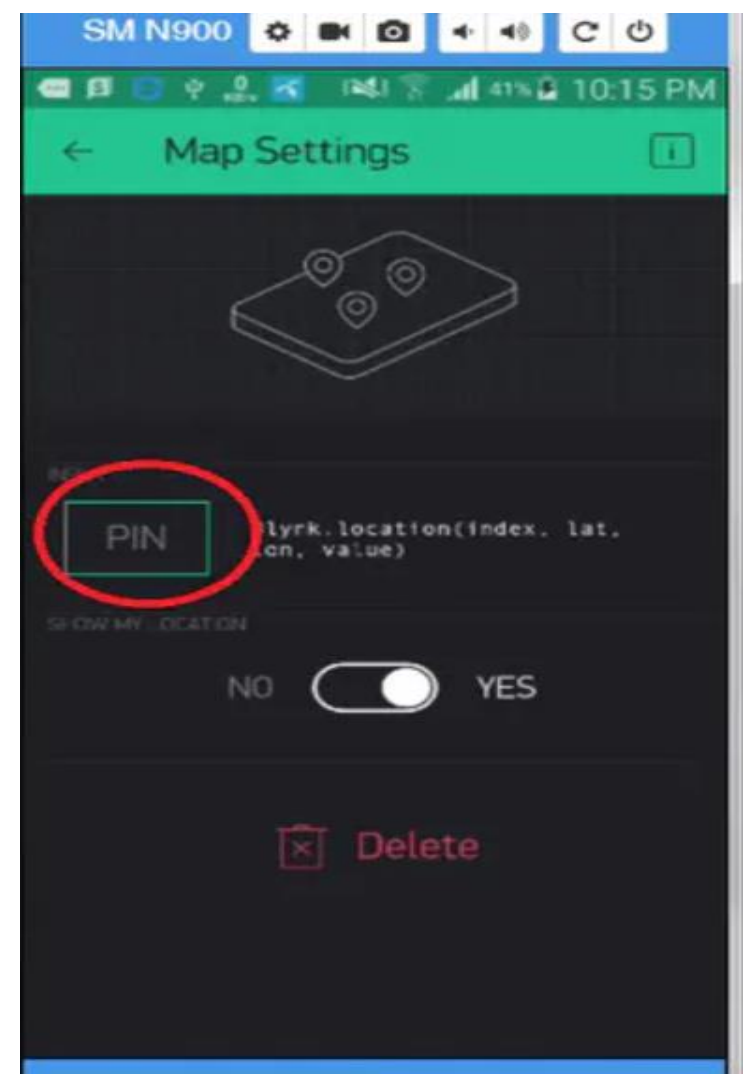

Fig. 12. Go to map setting

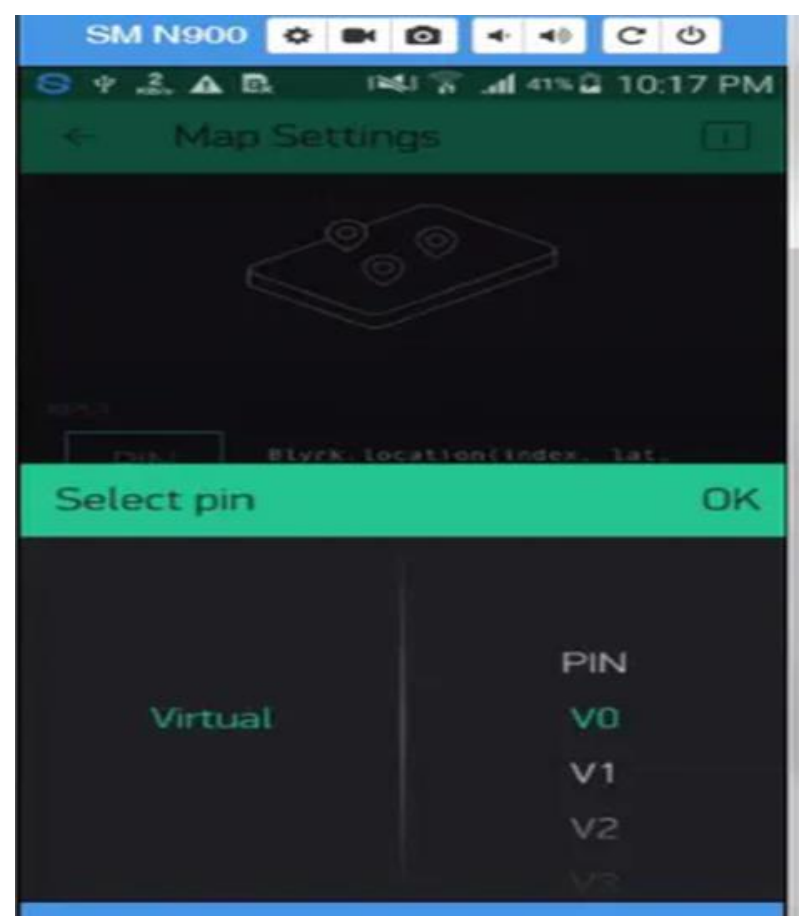

Fig. 13. Select Virtual Pin V0

\section{CONCLUSION}

The Android Application was built to give important data and follow the school transportation of the students. The framework is made from a worker and advanced cells. The framework can display its performance by following school transport from any place. Besides, our framework is very less expensive.

\section{FUTURE WORK}

We should foster an application for all ventures that utilize the transportation framework later. Other safety precautions must be taken. We also intend to install a live broadcast so that users can see what is going on inside the bus.

\section{REFERENCES}

1. R.C. Jisha, Aiswarya Jyothindranath, L Sajitha Kumary. "Iot based school bus tracking and arrival time prediction", 2017 International Conference on Advances in Computing, Communications and Informatics (ICACCI), 2017 Publication.

2. Shaaban K, BekKali A, Hamida EB, Kadri A. Smart Tracking system for school buses using passive RFID technology to enhance child safety. Journal of Traffic and Logistics Engineering. 2013 Dec;1(2):191-6. 
3. Arthur Thompson and Wayne Goodridge, "Bus Coming: Description and Evaluation of Bus Tracking System for Rural Areas", International Journal of Innovative Science, Engineering \& Technology, Vol. 1 Issue 5, July 2014.

4. Anon, Smart school bus monitoring and tracking system. IEEE Trans. Single processing, 55 (9), 200-205,2011.

5. Gunjal Sunil N., Joshi Ajinkya V., Gosavi Swapnil C., Kshirsagar Vyanktesh B, "Dynamic Bus Timetable Using GPS" International Journal of Advanced Research in Computer Engineering \& Technology (IJARCET), ISSN:2278-1323, Volume 3, Issue 3, March 2014.

6. Saifuzzaman M, Khan AH, Moon NN, Nur FN. Smart Security for an Organization based on IoT. International Journal of Computer Applications. 2017;165(10):33-8.

7. K. Sujatha, P. V. Nageswara Rao, K. J. Sruthi and A. A. Rao, "Design and development of android mobile based bus tracking system," 2014 First International Conference on Networks \& Soft Computing (ICNSC2014), 2014, pp. 231-235, doi: 10.1109/CNSC.2014.6906694.

8. M. N. Hasan and M. Sharif Hossen, "Development of An Android Based Real Time Bus Tracking System," 2019 1st International Conference on Advances in Science, Engineering and Robotics Technology (ICASERT), 2019, pp. 1-5, doi: 10.1109/ICASERT.2019.8934621.

9. S. Shah and B. Singh, "RFID based school bus tracking and security system," 2016 International Conference on Communication and Signal Processing (ICCSP), 2016, pp. 1481-1485, doi: 10.1109/ICCSP.2016.7754404. 